\title{
Development of Ventilatory Response to Transient Hypercapnia and Hypercapnic Hypoxia in Term Infants
}

\author{
SIGNE SØVIK AND KRISTIN LOSSIUS \\ Department of Physiology, Institute of Basic Medical Sciences, University of Oslo, NO-0317 Oslo [S.S.], \\ and Section of Neonatology, Department of Pediatrics, Rikshospitalet, NO-0027 Oslo [K.L.], Norway
}

\begin{abstract}
Whereas peripheral chemoreceptor oxygen sensitivity increases markedly after birth, previous studies of ventilatory responses to $\mathrm{CO}_{2}$ in term infants have shown no postnatal development. However, the hypercapnic challenges applied have usually been long-term, which meant that the effect of central chemoreceptors dominated. Oscillatory breathing, apneas, and sighs cause transient $\mathrm{PCO}_{2}$ changes, probably primarily stimulating peripheral chemoreceptors. We wanted to assess whether the immediate ventilatory responses to step changes in inspired $\mathrm{CO}_{2}$ and $\mathrm{O}_{2}$ in term infants undergo postnatal developmental changes. Twenty-six healthy term infants were studied during natural sleep $2 \mathrm{~d}$ and 8 wk postnatally. Ventilatory responses to a randomized sequence of $15 \mathrm{~s}$ hypercapnia $\left(3 \% \mathrm{CO}_{2}\right)$, hypoxia $\left(15 \% \mathrm{O}_{2}\right)$, and hypercapnic hypoxia $\left(3 \% \mathrm{CO}_{2}+15 \% \mathrm{O}_{2}\right)$ were recorded breath-by-breath using a pneumotachometer. Response rate, stimulus-response time, and response magnitude were analyzed with ANOVA after coherent averaging. Response rate increased with age by $30 \%$ (hypercapnia), 318\% (hypoxia), and $302 \%$ (hypercapnic hypoxia). Response rate during hypercapnic hypoxia exceeded rate during hypercapnia plus rate during hypoxia in wk 8 , but not on $\mathrm{d} 2$. Time to half-maximum response decreased by $3.4 \mathrm{~s}$ with age for the two hypercapnic stimuli but
\end{abstract}

\section{ABSTRACT}

was unchanged for hypoxia. Response magnitude was unchanged for hypercapnia, but increased for the two hypoxic stimuli. In conclusion, an interaction between the effects of hypercapnia and hypoxia on ventilatory response rate emerged between postnatal d 2 and wk 8 in term infants. Concomitantly, stimulus-response time to hypercapnic stimuli declined markedly. The development of a prompt response to transient hypercapnia may be important for infant respiratory stability. (Pediatr Res 55: 302-309, 2004)

Abbreviations
FiCO $_{2}$, fraction of inspired carbon dioxide
$\mathbf{f}_{\mathbf{R}}$, respiratory rate
PaCO $_{2}$, partial pressure of arterial carbon dioxide
$\mathbf{V}_{\mathbf{T}}$, tidal volume
$\dot{\mathbf{V}}_{\mathbf{E}}$, minute ventilation
$\dot{\mathbf{V}}_{\mathbf{E}}{ }_{\mathbf{4 5}}$, integrated ventilatory response for $45 \mathrm{~s}$ after the onset
of a 15 -s stimulus
$\dot{\mathbf{V}}_{\mathbf{E}}$ edian, median value of ventilation in the period $\pm 5 \mathrm{~s}$
from the end of a 15 -s stimulus
$\dot{\mathbf{V}}_{\mathbf{E}} \mathbf{m a x}$, maximum value of ventilation during a response,
determined after signal filtering

Peripheral and central chemoreceptors are crucial for the precise control of respiration. The carotid, aortic, and central chemoreceptors are functional even in fetal life $(1,2)$, but the transition to continuous breathing calls for rapid adjustments. The marked increase in arterial $\mathrm{Po}_{2}$ at birth brings about a corresponding resetting of carotid and aortic chemoreceptor $\mathrm{O}_{2}$ sensitivity, which takes place during the first postnatal days and weeks $(1,3-5)$. In contrast, studies of postnatal $\mathrm{CO}_{2}$ chemosensitivity show divergent results. Steady-state $\mathrm{CO}_{2}$ sensitivity increased after birth in some $(6-8)$ but not all species

Received October 15, 2002; accepted August 7, 2003.

Correspondence: Signe Søvik. M.D., Department of Anesthesiology, Aker University Hospital, Trondheimsveien 235, NO-0514 Oslo, Norway; e-mail: signe.sovik@basalmed.uio.no

Funded by the University of Oslo, Norway, with no extramural financial support.

DOI: 10.1203/01.PDR.0000106316.40213.DB
(9). Preterm infants displayed an age-related increase in steady-state $\mathrm{CO}_{2}$ sensitivity $(10,11)$, whereas term infants showed no such postnatal development $(12,13)$. Dynamic $\mathrm{CO}_{2}$ sensitivity was unchanged during the first month in neonatal lambs $(8,14)$, whereas in piglets the relative contribution of peripheral chemoreceptors to $\mathrm{CO}_{2}$ stimulation increased postnatally (15).

Ultimately, the combined effects of $\mathrm{PCO}_{2}$, arterial $\mathrm{Po}_{2}$, and $\mathrm{pH}$ determine the level of ventilation. Nonlinear interaction between $\mathrm{PCO}_{2}$ and $\mathrm{Po}_{2}$, i.e. increased $\mathrm{CO}_{2}$ chemosensitivity at increasing levels of hypoxia, has been described in carotid and aortic nerve recordings $(7,8,16)$ and in ventilatory responses in animals $(17,18)$ and adult humans $(19)$. Whether interaction between $\mathrm{PCO}_{2}$ and $\mathrm{PO}_{2}$ is present at birth and whether it increases postnatally differs from one species to another $(7,16$, 20). 
There have been few studies of infant respiratory control during transient hypercapnia, and to our knowledge, the hypercapnic-hypoxic ventilatory interaction has not been assessed in term infants. These are areas of interest because a fast-feedback system to maintain $\mathrm{PaCO}_{2}$ level is crucial for respiratory stability. Infants display a variety of oscillatory breathing patterns, sighs, and apneas, which produce marked but transient changes in $\mathrm{PaCO}_{2}$. Observed stimulus-response times indicate that the effect of peripheral chemoreceptors must dominate over that of central chemoreceptors in these situations $(17,21)$. The maturation of infant respiratory oscillations after a spontaneous sigh has been linked to peripheral chemoreceptor development (22), and it has been proposed that the emerging $\mathrm{CO}_{2}-\mathrm{O}_{2}$ interaction at the peripheral chemoreceptor level underlies the postnatal increase in hypoxic sensitivity (16).

We hypothesized that the peripheral chemoreceptors in healthy term infants undergo a postnatal increase in $\mathrm{CO}_{2}$ sensitivity, resulting in changes in the time course of ventilatory responses to step increases in $\mathrm{PCO}_{2}$. Secondly, we hypothesized that during simultaneous exposure, effects of hypercapnia and hypoxia on ventilation would interact, and that such interaction could develop postnatally.

\section{METHODS}

Subjects. Twenty-six randomly selected infants (16 girls) were studied at $2 \mathrm{~d}$ and $8 \mathrm{wk}$ postnatally. Inclusion criteria were healthy infant, birth weight $\geq 2500 \mathrm{~g}$, Apgar scores $\geq 8$ at 1 and $5 \mathrm{~min}$, spontaneous vaginal delivery to term, and uncomplicated pregnancy. Exclusion criteria were any chronic maternal illness and alcohol or illicit drug abuse. Median birth weight was $3482 \mathrm{~g}$ (range, 2940-4390), gestational age was 39 wk $6 \mathrm{~d}$ (range, 38 wk $0 \mathrm{~d}-41$ wk $2 \mathrm{~d}$ ), and maternal age was 30.2 y (range, 22.3-41.3 y). Fourteen women were nonsmokers, seven women smoked $\leq 5$ cigarettes/d, and five women smoked 10-20 cigarettes/d. Regional Ethics Committee approval and written informed consent from parents were obtained, and mothers were often present during experiments.

Procedures. Full details of this method of assessing chemoreceptor reflexes in infants have been published previously (23). The infants were studied during a daytime nap in a crib, sleeping in the supine position. Inspiratory gases were supplied through a lightweight, handheld face mask. Instantaneous switching between breathing gas mixtures of different composition was made possible by using computer-controlled electromagnetic valves. Gases were delivered dry at room temperature; the flow was $6.25 \mathrm{~L} / \mathrm{min}$. The $21-\mathrm{mL}$ face mask would thus be filled $0.2 \mathrm{~s}$ after a switch in delivered gas, whereas the functional dead space of the system, estimated at $1.5 \mathrm{~mL}$ (23), would be filled almost instantly.

Repeated chemoreceptor challenges were presented in a block randomized sequence during a full sleep period. The test gases were hypercapnia $\left(3 \% \mathrm{CO}_{2}\right.$ in $\left.21 \% \mathrm{O}_{2}\right)$, hypoxia $(15 \%$ $\left.\mathrm{O}_{2}\right)$, and hypercapnic hypoxia $\left(3 \% \mathrm{CO}_{2}\right.$ and $\left.15 \% \mathrm{O}_{2}\right)$. Each test gas period lasted $15 \mathrm{~s}$ and was followed by a 75-s period during which medical air was delivered in the mask.
$f_{R}, V T$, and $\dot{V}_{E}$ were calculated for each breath cycle from a pneumotachograph connected to the mask (23). Beat-by-beat heart rate was determined online from a three-lead ECG. Continuous measurements of $\mathrm{CO}_{2}$ and $\mathrm{O}_{2}$ immediately in front of the infants' nostrils were obtained by infrared spectroscopy and paramagnetic method, respectively (Artema Multigas Monitor MM201, Sundbyberg, Sweden; sampling rate 150 $\mathrm{mL} / \mathrm{min}$ ), and end-tidal values were estimated for each breath. Timed gas valve status was stored together with the physiologic data.

Data selection. Epochs of quiet sleep were selected on the basis of behavioral criteria. We discarded stimulation periods in which pneumotachograph recordings indicated that mask leakage had occurred, or in which a sigh occurred during the $20 \mathrm{~s}$ preceding stimulation.

Gas analysis measurements were inspected to ensure that inspired oxygen during hypoxic stimulation did not exceed $15 \%$ and that $\mathrm{FicO}_{2}$ during hypercapnic stimulation did not fall below 3\%. This would happen if peak inspiratory flow exceeded the flow of test gas, resulting in dilution of the stimulus with room air. Some accepted responses included a single breath cycle (an exaggerated breath or a sigh) in which such dilution did occur, but only test gas was inhaled in the ensuing breath cycles.

Ventilatory response categories. For each experiment and test gas type, each ventilatory response was categorized as being 1) smooth, with a gradual rise and fall, 2) spiked, i.e. a smooth response with one or two exaggerated breaths at its maximum phase, or 3) sighlike, in which the breathing pattern seemed unaffected until a sudden sigh was elicited.

Quantitative analysis. Ventilatory and end-tidal gas data were converted to $5-\mathrm{Hz}$ time series, and recordings in a time window from $50 \mathrm{~s}$ before to $150 \mathrm{~s}$ after the onset of each accepted stimulation period were aligned so that the onset of stimulus coincided before averaging. Time 0 was taken as the beginning of the first inspiration after a switch to test gas.

Coherently averaged $\dot{\mathrm{V}}_{\mathrm{E}}, \mathrm{V}$, and $\mathrm{f}_{\mathrm{R}}$ responses and the course of end-tidal $\mathrm{CO}_{2}$ and $\mathrm{O}_{2}$ values were calculated for each infant, age, and type of test gas. The averaged ventilatory responses were analyzed in a virtual instrument programmed in LabVIEW 5.0 (National Instruments Corp., Austin, TX, U.S.A.), as described previously (24). For illustration purposes, the coherently averaged $\dot{V}_{E}, V_{T}$, and $f_{R}$ response of the entire group was also calculated for each stimulus-age combination (Figs. 1 and 2).

Quantification of stimulus. The delivery of test gas from mask to lung was considered to be shown by the change in end-tidal $\mathrm{CO}_{2}$ and $\mathrm{O}_{2}$ values from baseline to $2 \mathrm{~s}$ after stimulus start, which is too early for any chemoresponse to have occurred. The ensuing rate of change in end-tidal $\mathrm{CO}_{2}$ and $\mathrm{O}_{2}$ (in kilopascals per second) was computed using linear regression for the interval $2-6 \mathrm{~s}$ after stimulus start. Maximum stimulus strength was taken as the change in end-tidal values from baseline to plateau level, i.e. the median value in the period $10-15 \mathrm{~s}$ after stimulus start.

The rate of $\dot{V}_{E}$ increase was estimated using linear regression during the interval 2-10 s after start of stimulation, a period when the response should result almost solely from stimulation 

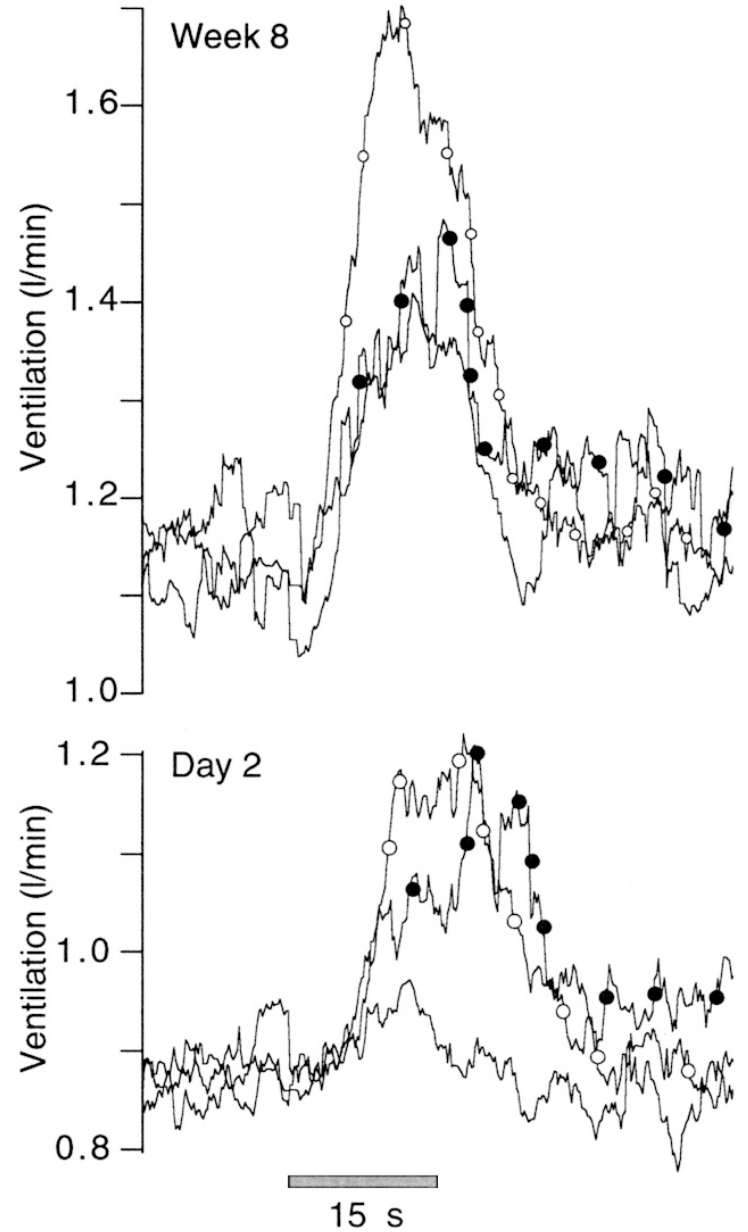

Figure 1. Coherently averaged ventilatory responses from 26 term infants. Hypercapnia (black circles), hypoxia (line only), and hypercapnic hypoxia (open circles) lasted $15 \mathrm{~s}$ (gray box). Ventilatory response delay was approximately $2 \mathrm{~s}$. Response rates increased with age, most markedly for hypoxia and hypercapnic hypoxia. Hypercapnic responses were slowest to reach halfmaximum value and remained longest above baseline, especially on $\mathrm{d} 2$. For all stimulus-age combinations, ventilation returned to prestimulus values well before the 20 -s baseline period of the next stimulation.

of the peripheral chemoreceptors $(21,25)$. Data from the initial $2 \mathrm{~s}$ of stimulation was omitted because lung-to-chemoreceptor transportation time makes it unlikely that chemoreceptor responses would occur in this time interval. Linear regression on data from a fixed time interval does not differentiate between an age-related increase in the steepness of the response slope and a reduction in response delay with an unaltered response slope. We nevertheless chose this method because the low number of breaths in the time interval of study would introduce considerable uncertainty if, for example, we used a polynomial curve fit.

Interaction of $\mathrm{CO}_{2}$ and $\mathrm{O}_{2}$ effects was assessed by subtracting the rates of ventilatory increases in response to hypercapnia and to hypoxia from the rate of response to hypercapnic hypoxia. If the effects are purely additive, this calculation will return a value close to zero. Interaction of effects will result in a positive value.

Ventilatory response magnitude was quantified by three measures: 1) $\dot{\mathrm{V}}_{\mathrm{E}}$ 45, 2) $\dot{\mathrm{V}}_{\mathrm{E}}$ median, and 3) $\dot{\mathrm{V}} \mathrm{Emax}$. The measures
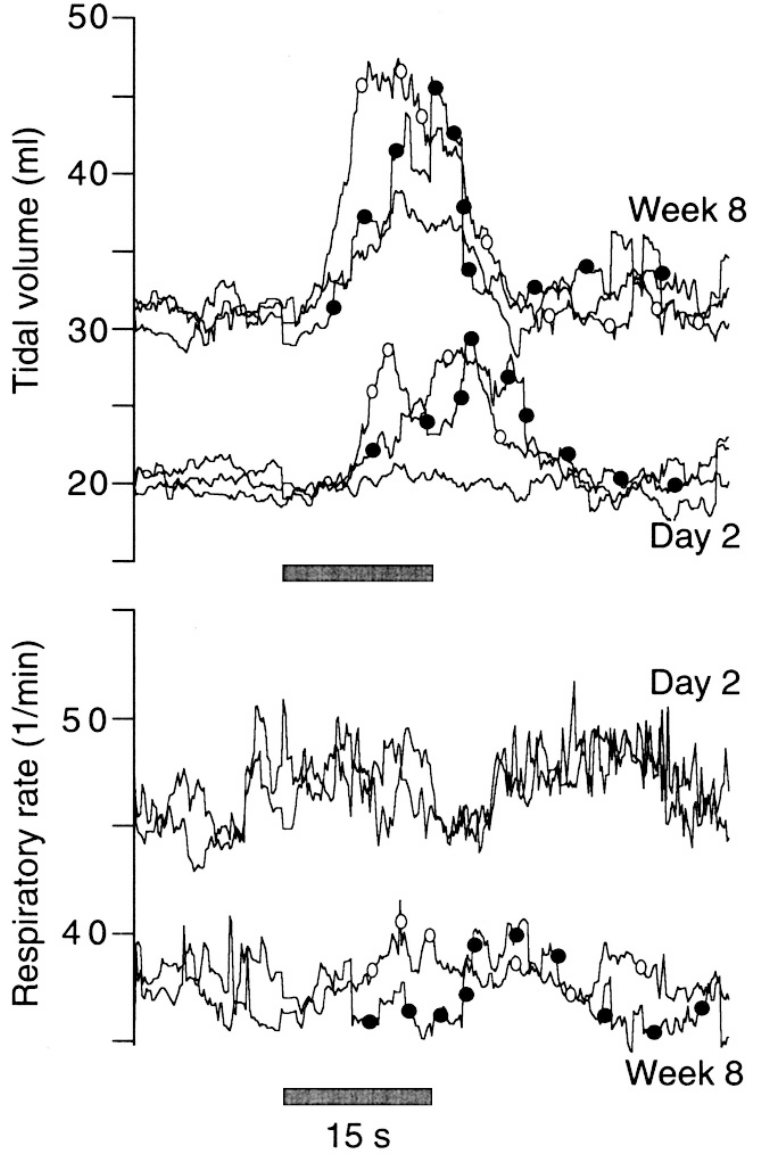

Figure 2. Coherently averaged tidal volume and respiratory rate responses from 26 term infants. Hypercapnia (black circles), hypoxia (line only), and hypercapnic hypoxia (open circles) lasted $15 \mathrm{~s}$ (gray box). Most of the $\dot{\mathrm{V}}_{\mathrm{E}}$ response was caused by an increase in VT. For hypercapnia and hypercapnic hypoxia, a reduction in stimulus-response time with increasing age was apparent. VT returned to baseline shortly after the end of stimulation. The $f_{R}$ response was more prolonged and contributed to the $\dot{\mathrm{V}}$ E response mainly on $\mathrm{d}$ 2 , when it was identical during hypercapnnia and hypercapnic hypoxia. A drop in $f_{R}$ at the time of maximum response signifies the frequent occurrence of sighs. The $f_{R}$ response to hypoxia was very small and is omitted for clarity.

of response magnitude were normalized with respect to the baseline value, i.e. the median value in the $20 \mathrm{~s}$ preceding stimulation.

Stimulus-response time was determined as the time from the onset of the first breath receiving test gas to the half-maximum $\dot{V}_{E}$ value. This point occurred on a steep part of the curve, making it a more robust measure than the onset of ventilatory increase or the time of maximum $\dot{\mathrm{V}}_{\mathrm{E}}$ response.

Before the maximum value and time-course of responses were assessed, high-frequency variation was removed from the signal with a 20-order low-pass Butterworth filter with a cutoff frequency of $0.20 \mathrm{~Hz}$. Time distortion of the signal was avoided by running the data forward and then backward again through the filter. The maximum values and the time at which they occurred in the resulting filtered responses were determined automatically, the search being limited to the first $30 \mathrm{~s}$ after the start of stimulation. The value halfway between the baseline and the maximum value was computed, and its time of occurrence on the filtered curve was found. Plots of the unfil- 
tered data and the filtered version with calculated time-points indicated were always inspected to ensure a sensible fit.

Statistical analysis. Values are medians with nonparametric near $95 \%$ confidence intervals, unless otherwise stated. For multiway analysis, we used a general mixed model of variance (BMDP 3V, release 7.0, 1993; BMDP Statistical Software Inc., Cork, Ireland), which uses maximum likelihood methods and allows repeated measures ANOVA to be applied to unbalanced data sets with missing observations. The magnitude and rate of change in end-tidal $\mathrm{O}_{2}$ and $\mathrm{CO}_{2}$ values for different test gas types and age groups were compared. The distribution of ventilatory response categories (smooth, spiked, or sighlike) was analyzed for effects of test gas type, postnatal age, and response magnitude expressed as $\dot{V}$ Emedian. The response rate and magnitude of ventilatory response (expressed as $\dot{V}_{E}{ }_{45}$, $\dot{V}$ Emedian, and Vंemax) were analyzed for effects of test gas type and postnatal age. Stimulus-response time was analyzed for effects of test gas type, postnatal age, and $\dot{V}_{E}$ response magnitude.

\section{RESULTS}

Successful recordings during quiet sleep were obtained in all 26 infants on $\mathrm{d} 2$ and in 20 of 26 infants in wk 8 (Table 1). The number of repeated responses obtained per experiment was similar for the three test gas types and was unrelated to age (Table 1). Mean $\dot{\mathrm{V}}_{\mathrm{E}}, \mathrm{V}_{\mathrm{T}}$, and heart rate during air breathing increased with age $(p<0.01)$, whereas $\mathrm{f}_{\mathrm{R}}$ decreased $(p<$ $0.02)$. Both $V_{T}$ and $f_{R}$ increased while test gases were being supplied ( $p<0.03$ for all comparisons; Fig. 2). VT increased more than $\mathrm{f}_{\mathrm{R}}$ when $\mathrm{CO}_{2}$-containing test gases were supplied but not during hypoxia ( $p<0.01$ at both ages).

Quantification of stimulus. The initial (2 s) decrease in end-tidal $\mathrm{PO}_{2}$ during hypoxia and increase in end-tidal $\mathrm{PCO}_{2}$ during hypercapnia were slightly larger on $\mathrm{d} 2$ than in wk 8 (Table 2; Mann-Whitney $U$ test; $p<0.05$ for both comparisons). A similar trend during hypercapnic hypoxia was not statistically significant.

The rate of fall in end-tidal $\mathrm{PO}_{2}$ during the second through sixth seconds of stimulation increased from d 2 to wk 8 , and the increase was similar for hypoxic and hypercapnic-hypoxic test gas $[0.27 \mathrm{kPa} / \mathrm{s}(0.25-0.32)$ on $\mathrm{d} 2$, and $0.34 \mathrm{kPa} / \mathrm{s}$

Table 1. Study age, baseline physiologic data, and responses

\begin{tabular}{lcc}
\hline & Session 1 & \multicolumn{1}{c}{ Session 2} \\
\hline Postnatal age (d) & $2.1(1.5-3.3)$ & $57.3(51.6-79.1)$ \\
Time since meal (min) & $60(10-150)$ & $90(20-170)$ \\
$\dot{\mathrm{V}}_{\text {baseline }}(\mathrm{L} / \mathrm{min})^{*}$ & $0.88(0.58-1.14)$ & $1.04(0.76-1.55)$ \\
$\mathrm{V}_{\mathrm{T} \text { baseline }}(\mathrm{mL})^{*}$ & $19.9(14.0-29.5)$ & $30.9(19.0-42.7)$ \\
$\mathrm{f}_{\mathrm{R} \text { baseline }}($ breaths $/ \mathrm{min}) \dagger$ & $44.9(28.8-63.2)$ & $37.0(29.9-51.5)$ \\
$\mathrm{HR}_{\text {baseline }}($ beats $/ \mathrm{min}) *$ & $110.3(83.4-141.0)$ & $123.0(106.1-148.7)$ \\
No. of responses§ to & & \\
$3 \% \mathrm{CO}_{2}$ & $4(1-9)$ & $4(1-7)$ \\
$15 \% \mathrm{O}_{2}$ & $4(2-10)$ & $5(0-8)$ \\
$3 \% \mathrm{CO}_{2}+15 \% \mathrm{O}_{2}$ & $4(2-9)$ & $4.5(1-8)$ \\
\hline
\end{tabular}

Values are medians and ranges. Difference between age groups: $* p<$ $0.001 ; \dagger p<0.02$, according to Mann-Whitney $U$ test. $\S$ Accepted responses to repeated test gas periods in individual experiments, i.e. the number of single responses underlying each infant's coherently averaged response.

Abbreviation: HR, heart rate.
(0.32-0.37) in wk 8; Mann-Whitney $U$ test, $p<0.02]$. The rate of rise of end-tidal $\mathrm{PCO}_{2}$ in the same time interval did not change with age and was similar for hypercapnic and hypercapnic-hypoxic test gas $[0.06 \mathrm{kPa} / \mathrm{s}$ (range, $0.05-0.07$ $\mathrm{kPa} / \mathrm{s})]$.

The baseline to plateau change in end-tidal values (Table 2) did not differ between age groups for any test gas. Hypoxia and hypercapnic hypoxia induced similar drops in end-tidal $\mathrm{PO}_{2}$, whereas end-tidal $\mathrm{PCO}_{2}$ increased slightly more during hypercapnia than during hypercapnic hypoxia (median difference, $0.13 \mathrm{kPa}$; Wilcoxon signed rank test $p<0.01$ ).

Ventilatory response type distribution. Ventilatory response type distribution differed according to test gas type and postnatal age at the time of study $(p=0.02)$, but was unaffected by the magnitude of the ventilatory response (V̈emedian). Age dependency was strong only for hypercapnic responses, during which a high frequency of spiked responses $(30 \%)$ declined by two thirds from $\mathrm{d} 2$ to $\mathrm{wk} 8(p=0.078)$, and sighlike responses declined from 5 to $1 \%(p=0.021)$, whereas smooth responses became more frequent $(p=0.007)$. Hypoxia caused spiked or sighlike responses least often $(12 \%)(p<0.035)$, whereas hypercapnic hypoxia frequently produced spiked responses $(24 \%)$ and more sighlike responses than the other stimuli $(p<$ 0.05 for both comparisons). A positive correlation was found between the fractions of spiked responses during hypercapnic hypoxia and those during hypercapnia $(p<0.001)$.

Rate of ventilatory increase-interaction of hypercapnia and hypoxia. Overall, the rate of $\dot{V}_{\mathrm{E}}$ response increased with age $(p<0.001)$. The magnitude of the age effect differed among test gas types $(p<0.001)$, the response rates to hypercapnia, hypoxia, and hypercapnic hypoxia increasing by $30 \%, 318 \%$, and $302 \%$, respectively (Table 3 ). The increase in the hypercapnic response rate was smaller because the rate was already relatively high on postnatal d 2, probably reflecting the high frequency of spiked responses.

The combined effect of hypercapnia and hypoxia on the rate of $\dot{V}_{E}$ increase was purely additive on $d 2$ (Fig. 3), the calculation rate during hypercapnic hypoxia minus the sum of rates during hypercapnia and during hypoxia returning a value that did not differ from zero $\left[0.18 \mathrm{~L} / \mathrm{min}^{2}\right.$ (range, -0.30 to 0.9 $\mathrm{L} / \mathrm{min}^{2}$; Wilcoxon signed rank sum test $p>0.20$ ]. In contrast, in wk 8 , the rate during hypercapnic hypoxia exceeded the sum of the rates during single stimuli by $2.22 \mathrm{~L} / \mathrm{min}^{2}$ (range, 1.08 to $\left.5.94 \mathrm{~L} / \mathrm{min}^{2} ; p<0.01\right)$. Thus, a more-than-additive interaction between the effects of hypercapnia and hypoxia on the initial rate of $\dot{V}_{E}$ increase had emerged.

Time course of ventilatory responses. Stimulus-response time, i.e. the time from onset of test gas to half-maximum $\dot{V}_{\mathrm{E}}$ response, depended on both test gas type and postnatal age (Fig. 4). On postnatal d 2, the stimulus-response time during hypercapnia was $2.3 \mathrm{~s}$ longer than during either hypercapnic hypoxia $(p=0.046)$ or hypoxia $(p=0.011)$. However, by postnatal wk 8 , the stimulus-response time during the two hypercapnic test gases had declined by $3.4 \mathrm{~s}$, whereas the response time during hypoxia was unchanged $(p=0.002)$. The difference in age effect between test gas types was significant regardless of whether sighlike responses were included or excluded from the analysis. In a multiple regression analysis of 
Table 2. Induced changes in end-tidal $\mathrm{PO}_{2}$ and $\mathrm{PCO}_{2}$ during stimulation

\begin{tabular}{|c|c|c|c|c|c|}
\hline \multirow[b]{2}{*}{ Gas } & \multirow[b]{2}{*}{ Age } & \multicolumn{2}{|c|}{ Change in end-tidal $\mathrm{Po}_{2}(\mathrm{kPa})$} & \multicolumn{2}{|c|}{ Change in end-tidal $\mathrm{PCO}_{2}(\mathrm{kPa})$} \\
\hline & & After $2 \mathrm{~s}$ & Plateau $\dagger$ & After $2 \mathrm{~s}$ & Plateau $\dagger$ \\
\hline Hypercapnic hypoxia & $\mathrm{d} 2$ & -2.37 & -4.29 & 0.85 & 1.24 \\
\hline \multirow[t]{2}{*}{ Hypoxia } & $\mathrm{d} 2$ & $-2.41^{*}$ & -4.41 & -0.02 & 0.01 \\
\hline & wk 8 & -2.08 & -4.34 & -0.01 & 0.08 \\
\hline Hypercapnia & d 2 & -0.12 & 0.42 & $1.03 *$ & 1.34 \\
\hline
\end{tabular}

Values are medians calculated from coherently averaged time courses of end-tidal $\mathrm{CO}_{2}$ and $\mathrm{O}_{2}$ in 26 infants.

* Difference between age groups by Mann-Whitney $U$ test $(p<0.05)$.

$\uparrow$ Change from baseline to median value in the interval $10-15 \mathrm{~s}$ after the start of a 15 -s test period.

Table 3. Ventilatory response rate and total ventilatory response to hypercapnic and hypoxic stimuli

\begin{tabular}{lccccc}
\hline & \multicolumn{2}{c}{ Ventilatory response rate $\left(\mathrm{L} / \mathrm{min}^{2}\right)$} & & \multicolumn{2}{c}{ Increase in ventilation, integrated during $45 \mathrm{~s}$} \\
$(\%)$
\end{tabular}

Data from 26 term infants. Values are group medians and nonparametric, near-95\% confidence intervals. Difference between age groups $* p=0.09, \uparrow p=$ 0.036. Response rate was calculated in the interval $2-10 \mathrm{~s}$ after the start of the 15 -s stimulus.

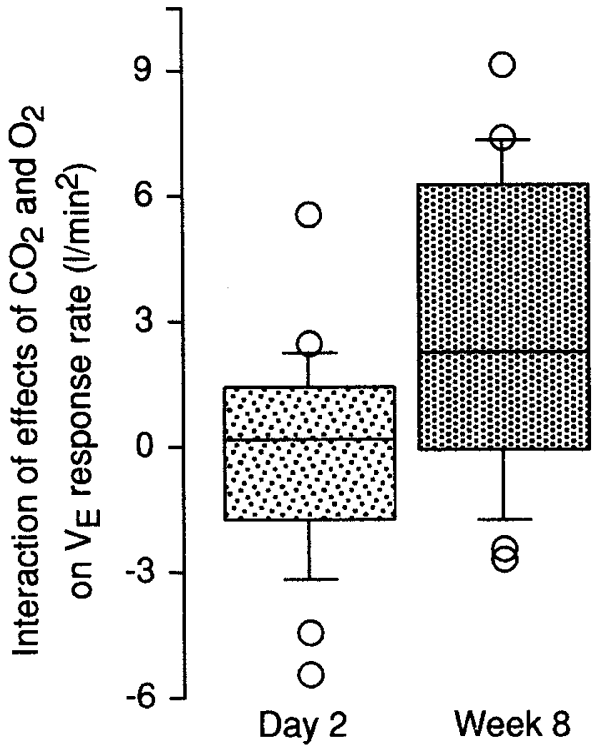

Figure 3. Emergence of more-than-additive interaction between effects of hypercapnia and hypoxia on rate of ventilatory increase in 26 term infants studied at two ages. Boxes comprise 25th to 75 th percentiles; 50 th percentiles (median values) are marked. Bars show 10th and 90th percentiles. The points represent individual values of response rates during hypercapnia and during hypoxia subtracted from the response rate during hypercapnic hypoxia. In contrast to findings on postnatal $\mathrm{d} 2$, the response rate during hypercapnic hypoxia clearly exceeded the sum of rates during hypercapnia and hypoxia in postnatal wk $8(p=0.005)$.

the effects of age, test gas type, $\dot{V}_{E}$ response rate, and $\dot{V}$ Emax on stimulus-response time, the magnitude of the regression coefficients indicated that the age-test gas type interaction was independently responsible for approximately two thirds of the decline in stimulus-response time.

Magnitude of ventilatory responses. The increase in median ventilation after $15 \mathrm{~s}$ of hypercapnic hypoxia (نंEmedian) was larger than the sum of $\dot{V}$ emedian during hypercapnia and VEmedian during hypoxia, at both ages studied (Wilcoxon

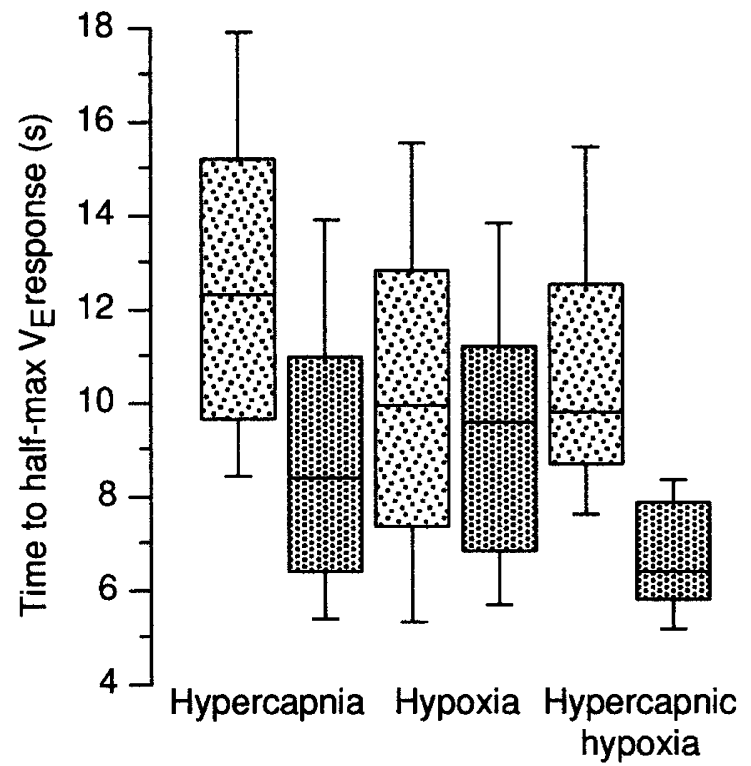

Figure 4. Time from stimulus start to half-maximum (half-max) VंE response to hypercapnia, hypoxia, and hypercapnic hypoxia in 26 infants on postnatal d 2 (light gray) and in wk 8 (dark gray). Values were calculated in low-pass filtered coherently averaged responses from individual infants. Time to halfmaximum response was longest during hypercapnia on $\mathrm{d} 2$. By wk 8 it had decreased by $3.4 \mathrm{~s}$ for hypercapnia and hypercapnic hypoxia alike, whereas hypoxic response timing remained unchanged.

signed rank sum test; $p<0.01$; Fig. 5). Thus, an interaction of effects of hypercapnia and hypoxia on early response magnitude was present. In contrast, the total ventilatory increase $\left(\dot{V}_{\mathrm{E}}\right.$ 45 ) in response to hypercapnic hypoxia equaled the sum of the total ventilatory increases to hypercapnia and to hypoxia (Table 3). With increasing age, the total response to hypercapnic hypoxia increased $(p=0.036)$, the total response to hypoxia tended to increase $(p=0.09)$, and the total response to hypercapnia was unchanged (Table 3). Neither Vंemedian nor $\dot{V}$ Emax during hypercapnic hypoxia showed significant 


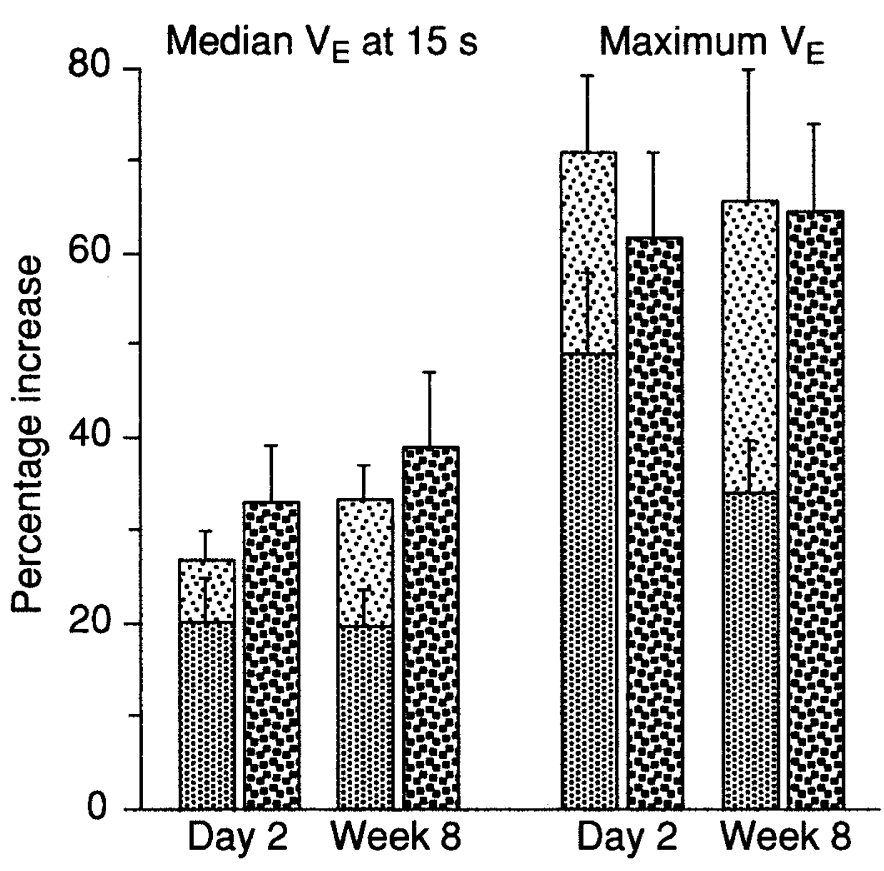

Figure 5. Magnitude of ventilatory response to hypercapnia (gray), hypoxia (light scatter), and hypercapnic hypoxia (heavy scatter) at two ages. Columns show means, error bars show 95\% confidence intervals. Responses to hypercapnia and to hypoxia are stacked to facilitate comparison with response to hypercapnic hypoxia. The $\dot{V}$ Emedian value around the end of the 15-s stimulation increased with age for hypoxia and remained unchanged for hypercapnia. A positive interaction of effects of hypoxia and hypercapnia was present at both ages; VEmedian for hypercapnic hypoxia exceeded the sum of responses to hypercapnia and to hypoxia by $4.1 \%$ (range, $1.0-10 \% ; p<0.01$ ). $\dot{V}$ Emax value increased with age for hypoxia and tended to decrease for hypercapnia, but there was no significant change for hypercapnic hypoxia and no detectable interaction.

changes with age (Fig. 5). Underlying this finding were agerelated increases in both Vंemedian $(p=0.005)$ and $\dot{V}$ Emax $(p$ $=0.043$ ) for hypoxic responses, but no change in $\dot{V}$ emedian and a downward trend in $\dot{V}$ Emax for hypercapnic responses.

\section{DISCUSSION}

The main findings in this study of ventilatory responses in healthy term infants were 1) a more-than-additive interaction of hypercapnic-hypoxic effects on ventilatory response rate emerging during the first two postnatal months, and 2) a marked age-related decrease in stimulus-response time during hypercapnic stimuli but not during hypoxia. Taken together, these could suggest a maturation of peripheral $\mathrm{CO}_{2}$ chemoreception.

Methodological considerations. This study was designed to assess the combined respiratory effects of hypercapnia and hypoxia. Stimulus duration and analytical approach were selected to differentiate as clearly as possible between the effects of peripheral and central chemoreceptor stimulation. After carotid chemoreceptor denervation, neonatal lambs exposed to a step increase in $\mathrm{FiCO}_{2}$ showed no ventilatory response during the first $10 \mathrm{~s}$ of stimulation $(21,25)$, and the (central) ventilatory response was markedly reduced for approximately $25 \mathrm{~s}$ (21). In our study, the measures of response magnitude and the time to half-maximum response reflected both peripheral and central chemoreceptor effects. In contrast, ventilatory response rate was calculated from the initial $10 \mathrm{~s}$ of the test period, when it should be determined almost exclusively by peripheral chemoreceptors.

Age-related changes in infant respiratory pattern and mechanics may confound the interpretation when chemoreceptor characteristics are inferred from respiratory responses to changes in fraction of inspired oxygen and carbon dioxide. Evaluating the strength and time profile of stimuli through end-tidal gas values, we found the initial ( $2 \mathrm{~s}$ ) hypoxia and hypercapnia to be slightly greater on postnatal $\mathrm{d} 2$ than in wk 8, although plateau stimulation strength was similar. Alveolar ventilation per body weight was probably similar at the two ages, but the younger infants may have had lower functional residual lung capacity, giving a shorter equilibration time after a switch of gas type. The early rate of change in end-tidal values increased with age for $\mathrm{P}_{2}$ but was unchanged for $\mathrm{PCO}_{2}$, and neither depended on whether stimuli were given alone or in combination. Overall, it could not be demonstrated that the delivery of test gas to the pulmonary circulation was faster or more complete in older infants or during steeper or larger ventilatory responses.

Upper and lower airway reflexes probably modified the $\dot{V}_{E}$ responses. The spiked responses might represent what is called Head's paradoxical inflation-augmenting reflex, in which a VT increase itself triggers an unusually large breath cycle. Sighlike responses, most frequent during hypercapnia on postnatal d 2 , could be the result of stimulation of laryngeal $\mathrm{CO}_{2}$ receptors initiating the first step of arousal (26).

Development of ventilatory chemoreceptor responses. For hypercapnic responses, both the median value after $15 \mathrm{~s}$ of stimulation and the total integrated response were identical on d 2 and in wk 8 (Fig. 5 and Table 3). Thus, as early as the immediate neonatal period, the combined action of peripheral and central chemoreceptors closely controls $\mathrm{PaCO}_{2}$ and thus $\mathrm{pH}$ during transient mild hypercapnia. However, in 8-wk-old infants, the time needed for $\dot{V}_{\mathrm{E}}$ to return to baseline level after stimulation was shorter (Fig. 1), possibly reflecting a higher degree of peripheral handling of the $\mathrm{CO}_{2}$ challenge. As expected, the magnitude of response to hypoxia increased with age, and the magnitude of hypercapnic-hypoxic responses increased accordingly.

Interaction of the effects of hypercapnia and hypoxia on the

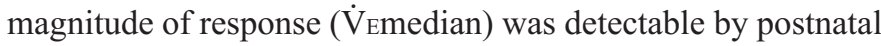
d 2 (Fig. 5). For the $\dot{V}_{E}$ response rate, $\mathrm{CO}_{2}-\mathrm{O}_{2}$ interaction emerged between d 2 and wk 8 (Figs. 1 and 3 and Table 3). Although a noninvasive study does not make it possible to rule out central nervous contribution, the developing more-thanadditive effect of $\mathrm{CO}_{2}$ and $\mathrm{O}_{2}$ was demonstrated for a variable for which it is likely that peripheral chemoreceptors were mainly responsible. A positive interaction between $\mathrm{CO}_{2}$ and $\mathrm{O}_{2}$ has been described at the peripheral chemoreceptor level in several species $(7,8,16,27)$. Moreover, selective perfusion studies in awake goats (28) and studies in piglets (17) and adult humans (29) indicate that the hypercapnic-hypoxic ventilatory interaction is mediated largely by the peripheral chemoreceptors, with only an additive effect of central $\mathrm{CO}_{2}$ chemoreception. 
Stimulus-response time was estimated by the time taken to reach the half-maximum $\dot{V}_{E}$ value. It would therefore increase with mask-to-lung-to-receptor transportation time, receptor and central nervous processing time, and value of maximum $\dot{V}_{E}$ response. A high $\dot{V}_{E}$ response rate would decrease stimulus-response time.

We found a $3.4 \mathrm{~s}$ decline in stimulus-response time with postnatal age for the two hypercapnic stimuli, but none for hypoxia. Regression coefficients indicated that approximately two thirds of this decline was independent of changes in $\dot{V}_{E}$ max and $\dot{V}_{E}$ response rate; reduced central nervous processing time is unlikely to produce a change of this magnitude. No age-related reduction in mask-to-lung transportation time could be demonstrated, and in any case, this, like an age-related increase in cardiac output, would also have affected hypoxic responses. Thus, the isolated decline in stimulus-response time for hypercapnic responses is more readily explained by an increase in the contribution of peripheral $\mathrm{CO}_{2}$ chemoreceptors relative to the central $\mathrm{CO}_{2}$ chemoreceptors.

In neonatal piglets exposed to a square-wave increase in end-tidal $\mathrm{CO}_{2}$, the ratio of peripheral to total $\mathrm{CO}_{2}$ chemosensitivity increased postnatally $(15,30)$. The estimated difference between peripheral and central chemoreceptors in lung-toreceptor transportation times was $2 \mathrm{~s}$, and the peripheral $\dot{\mathrm{V}}_{\mathrm{E}}$ chemoresponse was extremely steep compared with the central response (estimated time constants were $1-3 \mathrm{~s}$ versus $60-70 \mathrm{~s}$ ). Thus, an age-related increase in peripheral $\mathrm{CO}_{2}$ chemosensitivity could have produced a reduction in the time taken to achieve half-maximum hypercapnic response of the magnitude observed in our study.

The timing of development cannot be determined exactly in a study with only two points of observation. However, in a previous study of 14 term infants studied $2 \mathrm{~d}$ and $4 \mathrm{wk}$ postnatally (24), we found a decline in stimulus-response time to hypercapnia identical to that found in the present study (3.3 $\mathrm{s}$ versus $3.4 \mathrm{~s}$; unpublished data). Thus, infant $\mathrm{CO}_{2}$ chemosensitivity appears to undergo development during the first postnatal month. In various animal studies, the maturation of peripheral $\mathrm{CO}_{2}$ chemosensitivity and $\mathrm{CO}_{2}-\mathrm{O}_{2}$ interaction occurred from a few days to several months postnatally ( 7 , $14-16,20,30)$.

It has been suggested that $\mathrm{CO}_{2}-\mathrm{O}_{2}$ interaction at the level of the peripheral chemoreceptor or its innervation underlies the postnatal increase in $\mathrm{O}_{2}$ sensitivity (16). In the present study, interaction between $\mathrm{CO}_{2}$ and $\mathrm{O}_{2}$ effects on the magnitude of the ventilatory response was present $36-79 \mathrm{~h}$ after birth, at a time when hyperoxic responses are clearly detectable (4). Moreover, interaction between $\mathrm{CO}_{2}$ and $\mathrm{O}_{2}$ effects on the $\dot{\mathrm{V}}_{\mathrm{E}}$ response rate emerged over the following weeks, a long-term development comparable to that of the hyperoxic responses observed in a previous study (4). However, several mechanisms may be responsible for the resetting of $\mathrm{O}_{2}$ sensitivity. Further developmental studies, functional and biochemical and with a high "time resolution," are warranted to clarify this matter.

\section{CONCLUSIONS}

In quietly sleeping healthy term infants, we found that the overall ventilatory handling of a hypercapnic challenge was fully functional on postnatal $\mathrm{d} 2$, and $\mathrm{CO}_{2}$ and $\mathrm{O}_{2}$ interacted to increase the magnitude of the ventilatory response. However, during the following $8 \mathrm{wk}$, the ventilatory stimulus-response time to hypercapnic stimuli declined markedly, whereas a more-than-additive interaction between $\mathrm{CO}_{2}$ and $\mathrm{O}_{2}$ effects on ventilatory response rate emerged. We suggest that a developmental increase in the contribution of peripheral $\mathrm{CO}_{2}$ chemoreceptors relative to the central $\mathrm{CO}_{2}$ chemoreceptors may underlie our findings. Improved ability to detect and respond to transient changes in $\mathrm{PCO}_{2}$ is important for infant respiratory stability, and may contribute to the age-related changes in breathing pattern seen in developing infants.

Acknowledgment. The authors thank Professor Lars Walløe, M.D., Ph.D., for fruitful discussions on our analytical approach and a critical review of the manuscript.

\section{REFERENCES}

1. Blanco CE, Dawes GS, Hanson MA, McCooke HB 1984 The response to hypoxia of arterial chemoreceptors in fetal sheep and newborn lambs. J Physiol (Lond) $351: 25-37$

2. Hilaire G, Duron B 1999 Maturation of the mammalian respiratory system. Physiol Rev 79:325-360

3. Kumar P, Hanson MA 1989 Re-setting of the hypoxic sensitivity of aortic chemoreceptors in the newborn lamb. J Dev Physiol 11:199-206

4. Søvik S, Lossius K, Eriksen M, Grøgaard J, Walløe L 1999 Development of oxygen sensitivity in infants of smoking and non-smoking mothers. Early Hum Dev 56:217232

5. Gauda EB, Lawson EE 2000 Developmental influences on carotid body responses to hypoxia. Respir Physiol 121:199-208

6. Guthrie RD, Standaert TA, Hodson WA, Woodrum DE 1981 Development of $\mathrm{CO}_{2}$ sensitivity: effects of gestational age, postnatal age, and sleep state. J Appl Physiol 50:956-961

7. Carroll JL, Bamford OS, Fitzgerald RS 1993 Postnatal maturation of carotid chemoreceptor responses to $\mathrm{O}_{2}$ and $\mathrm{CO}_{2}$ in the cat. J Appl Physiol 75:2383-2391

8. Calder NA, Kumar P, Hanson MA 1997 Development of carotid chemoreceptor dynamic and steady-state sensitivity to $\mathrm{CO}_{2}$ in the newborn lamb. J Physiol (Lond) 503:187-194

9. Haxhiu-Poskurica B, Carlo WA, Miller MJ, DiFiore JM, Haxhiu MA, Martin RJ 1991 Maturation of respiratory reflex responses in the piglet. J Appl Physiol 70:608-616

10. Rigatto H, Brady JP, de la Torre Verduzco R 1975 Chemoreceptor reflexes in preterm infants: II. The effect of gestational and postnatal age on the ventilatory response to inhaled carbon dioxide. Pediatrics 55:614-620

11. Frantz ID, Adler SM, Thach BT, Taeusch Jr HW 1976 Maturational effects on respiratory responses to carbon dioxide in premature infants. J Appl Physiol 41:41-45

12. Haddad GG, Leistner HL, Epstein RA, Epstein MA, Grodin WK, Mellins RB 1980 $\mathrm{CO}_{2}$-induced changes in ventilation and ventilatory pattern in normal sleeping infants. J Appl Physiol 48:684-688

13. Schafer T, Schafer D, Schlafke ME 1993 Breathing, transcutaneous blood gases, and $\mathrm{CO}_{2}$ response in SIDS siblings and control infants during sleep. J Appl Physiol $74: 88-102$

14. Canet E, Kianicka I, Praud JP 1996 Postnatal maturation of peripheral chemoreceptor ventilatory response to $\mathrm{O}_{2}$ and $\mathrm{CO}_{2}$ in newborn lambs. J Appl Physiol 80:1928-1933

15. Wolsink JG, Berkenbosch A, DeGoede J, Olievier CN 1993 Maturation of the ventilatory response to $\mathrm{CO}_{2}$ in the newborn piglet. Pediatr Res 34:485-489

16. Pepper DR, Landauer RC, Kumar P 1995 Postnatal development of $\mathrm{CO}_{2}-\mathrm{O}_{2}$ interaction in the rat carotid body in vitro. J Physiol (Lond) 485:531-541

17. Wolsink JG, Berkenbosch A, DeGoede J, Olievier CN 1992 The effects of hypoxia on the ventilatory response to sudden changes in $\mathrm{CO}_{2}$ in newborn piglets. J Physiol (Lond) 456:39-48

18. Daristotle L, Berssenbrugge AD, Bisgard GE 1987 Hypoxic-hypercapnic ventilatory interaction at the carotid body of awake goats. Respir Physiol 70:63-72

19. Masuda A, Ohyabu Y, Kobayashi T, Yoshino C, Sakakibara Y, Komatsu T, Honda Y 2001 Lack of positive interaction between $\mathrm{CO}_{2}$ and hypoxic stimulation for $\mathrm{P}\left(\mathrm{CO}_{2}\right)$-VAS response slope in humans. Respir Physiol 126:173-181

20. Wolsink JG, Berkenbosch A, DeGoede J, Olievier CN 1994 Ventilatory interaction between hypoxia and hypercapnia in piglets shortly after birth. Respir Physiol $96: 25-35$ 
21. Carroll JL, Canet E, Bureau MA 1991 Dynamic ventilatory responses to $\mathrm{CO}_{2}$ in the awake lamb: role of the carotid chemoreceptors. J Appl Physiol 71:2198-2205

22. Fleming PJ, Goncalves AL, Levine MR, Woollard S 1984 The development of stability of respiration in human infants: changes in ventilatory responses to spontaneous sighs. J Physiol (Lond) 347:1-16

23. Søvik S, Eriksen M, Lossius K, Grøgaard J, Walløe L 1999 A method of assessing ventilatory responses to chemoreceptor stimulation in infants. Acta Paediatr 88:563-570

24. Søvik S, Lossius K, Walløe L 2001 Heart rate response to transient chemorecepto stimulation in term infants is modified by exposure to maternal smoking. Pediatr Res 49:558-565

25. Carroll JL, Bureau MA 1988 Peripheral chemoreceptor $\mathrm{CO}_{2}$ response during hyperoxia in the 14-day-old awake lamb. Respir Physiol 73:339-349
26. Lijowska AS, Reed NW, Chiodini BA, Thach BT 1997 Sequential arousal and airway-defensive behavior of infants in asphyxial sleep environments. J Appl Physio 83:219-228

27. Lahiri S, Mulligan E, Nishino T, Mokashi A 1979 Aortic body chemoreceptor responses to changes in $\mathrm{PCO}_{2}$ and $\mathrm{PO}_{2}$ in the cat. J Appl Physiol 47:858 -866

28. Daristotle L, Bisgard GE 1989 Central-peripheral chemoreceptor ventilatory interaction in awake goats. Respir Physiol 76:383-391

29. Yang F, Khoo MC 1994 Ventilatory response to randomly modulated hypercapnia and hypoxia in humans. J Appl Physiol 76:2216-2223

30. Wolsink JG, Berkenbosch A, DeGoede J, Olievier CN 1991 Ventilatory sensitivities of peripheral and central chemoreceptors of young piglets to inhalation of $\mathrm{CO}_{2}$ in air. Pediatr Res 30:491-495 